\title{
Developing a probabilistic tool for assessing the risk of overheating in buildings for future climates
}

\author{
David P. Jenkins ${ }^{1, *}$, Sandhya Patidar ${ }^{2}$, Phil Banfill ${ }^{1}$, Gavin Gibson ${ }^{2}$ \\ ${ }^{1}$ Urban Energy Research Group, School of Built Environment, Heriot-Watt University, Edinburgh, UK \\ ${ }^{2}$ School of Mathematical and Computer Sciences (address as above) \\ *Corresponding author.Tel: +44 (0)1314514637,E-mail:D.P.Jenkins@hw.ac.uk
}

\begin{abstract}
The effect of projected climate change on building performance is currently a growing research area. Building designers and architects are becoming more concerned that buildings designed for the current climate might not provide adequate working and living environments in the coming decades. Advice is needed to guide how existing buildings might be adapted to cope with this future climate, as well as guidance for new building design to reduce the chances of the building failing in the future. The Low Carbon Futures Project, as part of the Adaptation and Resilience to Climate Change (ARCC) programme in the UK, is looking at methods of integrating the latest climate projections from the UK Climate Impact Programme (UKCIP) into building simulation procedures. The main obstacle to this objective is that these projections are probabilistic in nature; potentially thousands of equally-probably climate-years can be constructed that describe just a single scenario. The project is therefore developing a surrogate procedure that will use regression techniques to assimilate this breadth of climate information into the building simulation process.
\end{abstract}

Keywords: Climate change, Building simulation, Overheating

\section{Nomenclature}

$T_{o}(t)$ internal air temperature at hour t.......... ${ }^{\circ} \mathrm{C}$

$\theta_{0}$ regression constants ......

$m_{j}$ regression coefficients.....

$\chi_{j}(t)$ hourly climate parameter

\section{Introduction}

In the UK, for both new build and building refurbishment sectors, legislation is currently being discussed to achieve low-carbon buildings through the use of new design and technologies [1]. It is therefore inevitable that a level of uncertainty exists with regards to the future energy performance of such buildings. In addition to this, with future climate warming being predicted over the coming decades for the UK, there is an uncertainty with regards to the comfort performance of such buildings - will future climate warming negate certain design assumptions for buildings designed or retrofitted for a current climate? For a naturally ventilated building this might mean internal temperatures exceeding design thresholds for significant periods of the year, whereas a mechanically cooled building might be operating with an under-sized cooling plant. This describes the problem that the concept of "adaptation" is trying to solve; what changes to our current approach should be taken now to ensure a building will maintain adequate levels of thermal comfort in the future? Such an analysis requires a suitable form of future climate projections, which themselves are inherently uncertain. Previous approaches to climate projections have been deterministic [2], in that they specify an estimated value of expected climate change for a specific scenario. The most recent UK Climate Projections (UKCP'09[3]) from the UK Climate Impact Programme (UKCIP) takes a different approach, with climate projections provided in a probabilistic form. These have been constructed from multiple iterations of climate models, which have undergone a degree of downscaling by geography and temporal resolution. The result of this can be many thousands of possible climate files describing just a single future scenario (see section 2). If such information is to be incorporated into building design approaches, it is clear that an 
additional step is required which can either simplify this climate information or provide an algorithm which processes this data in a way that might be useful for a building designer. If this goal can be achieved, then the result will be a method for incorporating the uncertainty of climate projections into building design and allowing the designer to choose adaptation options (such as shading or ventilation techniques) which will give a high probability of adequate thermal comfort in that building for a future climate. This work forms part of the Low Carbon Futures project, sponsored by the Adaptation and Resilience in a Changing Climate (ARCC) Programme [4].

\section{Methodology}

The following section will describe the approach being taken by the Low Carbon Futures project, which includes obtaining weather data, carrying out extensive building simulation and then using the obtained relationships to construct a regression relationship between climate and internal temperatures of a building.

\subsection{Weather Generator}

The Low Carbon Futures project [5] obtains future climate projections from the UKCP'09 Weather Generator. This can provide a number of statistically equivalent 30-year time series projections which describe a specific future scenario (e.g. low-emission, 2020-2049) for a specific location (based on a grid map of the UK). The weather variables can be generated at monthly, daily or hourly scales and include: total hourly precipitation (mm), mean hourly temperature $\left({ }^{\circ} \mathrm{C}\right)$, vapour pressure (hPA), relative humidity $(\%)$, sunshine fraction (of an hour), downward diffuse radiation and direct radiation (both $\mathrm{W} / \mathrm{m}^{2}$ ). If the user is downloading 100 time-series (the maximum allowed for each iteration), each run will produce 3000 (30years x 100 files) equally probable climate years at an hourly resolution. The resulting climate information can therefore be vast in scale. If using building simulation, the options might be to either i) choose just one (or a small number) of these representative climate-years to simulate a building with or ii) provide a short-cut or emulation step to make the building simulation process itself, over many climates, more efficient. The Low Carbon Futures project is investigating the latter approach. The timelines looked at for this study will be 2020-2049, 2040-2069 and 2060-2089 (referred to as "2030s", "2050s" and "2080s" respectively). The Weather Generator provides three future emission scenarios, namely "low", "medium” and "high" (as defined by UKCP'09), all of which will be included in the analysis. With two locations currently being investigated by the project (Edinburgh and London), this provides a total of 20 climate scenarios, including a baseline "current" climate (from 19601990 data) in both locations.

\subsection{Building Simulation}

While the described approach could be used with any building simulation software, the project uses ESP-r, an open-source package. To adequately describe internal temperature profiles (and therefore provide useful information with regards to overheating metrics), it is important to carry out these simulations with dynamic simulation software and at a suitable temporal resolution (in this case hourly). Such software will allow for the thermal response of the building over time to be suitably expressed, as well as providing a method for defining various adaptation scenarios. A range of buildings are being simulated by the project, some taken from real case-studies while others are adopted from previous simulation studies such as the Tarbase project [6]. This paper overviews two of these buildings: a standard dwelling [7] and a primary school [8], both of which are naturally ventilated. Different buildings have different overheating definitions and might have specific adaptation options that are related to 
the occupancy and construction characteristics. It is therefore important, when assessing overheating risks through simulation, to have a method that can be used for a range of buildings and a range of overheating metrics. The hypothesis of the project is that one initial simulation can identify the relationship between an hourly climate file and hourly internal temperatures of a building, summarised by an appropriate regression equation. This regression equation can then be used for a vast array of climates, without the designer having to resort back to full building simulations for all the other climates. This methodology is seen as an acceptable compromise between maintaining the detailed calculation of dynamic simulation software (as such a calculation will be required to start the process), while providing a means to achieving the equivalent of (up to) thousands of climate inputs through a given building model.

For validation purposes, each building in the project is simulated for a total of 2000 climateyears, encompassing the described range of emission scenarios, timelines and locations. A script has been developed for the project (by University of Strathclyde, who develop ESP-r) that allows many climates to be simulated in succession. While this is still a time-consuming process (and would not be practical for use in industry), it allows the project team to carry out a validation exercise across a large number of climates. For each iteration, the hourly internal temperature profile for the entire year is recorded for use in the regression exercise.

\subsection{Validation of regression analysis}

Following the building simulations, a large database of hourly climate metrics and hourly internal temperatures (by zone in the building, where the user may choose to focus on the area of the building that is most prone to overheating or has the highest occupancy) is created. The next step is to demonstrate a statistical relationship between the climate projections and the resulting building temperatures. A regression equation can be formulated describing this that is calibrated using just one climate, i.e. calculating the appropriate regression coefficients, and this same relationship can be used for all the other climates to investigate whether the same relationship will hold for that building. A simple regression equation will have a large number of terms; not only should the range of influential climate metrics be included (listed in section 2.1) for a given hour, but also the same metrics for previous hours, due to the dynamic thermal response of a building to such changes over time. It is found that the regression equation can emulate simulation results if climate information from the previous 72 hours is included. Taken across seven different climate metrics this potentially provides 504 terms in the regression equation (i.e. $72 \times 7=504$ ). However, using the established statistical technique of Principal Component Analysis (PCA), these terms can be reduced to just 33 (this is discussed in detail elsewhere [9]). The resulting regression equation is then of the form:

$$
T_{0}(t)=\theta_{0}+\sum_{j=1}^{33} m_{j} X_{j}(t)
$$

For adaptation scenarios (e.g. a physical change to the building that might combat overheating), Eqn. (1) can either be recalibrated following another simulation or a series of correction equations applied that are specific to those adaptation choices [9].

The results of the validation exercise, comparing hourly internal temperatures from the calibrated regression equation to that of ESP-r simulation, are demonstrated in Fig. 1. This is for the case of a domestic building, without any adaptation measures and with 100 climateyears representing a London 2030, medium emission scenario (though similar results have been obtained from other buildings and adaptation and climate scenarios). 


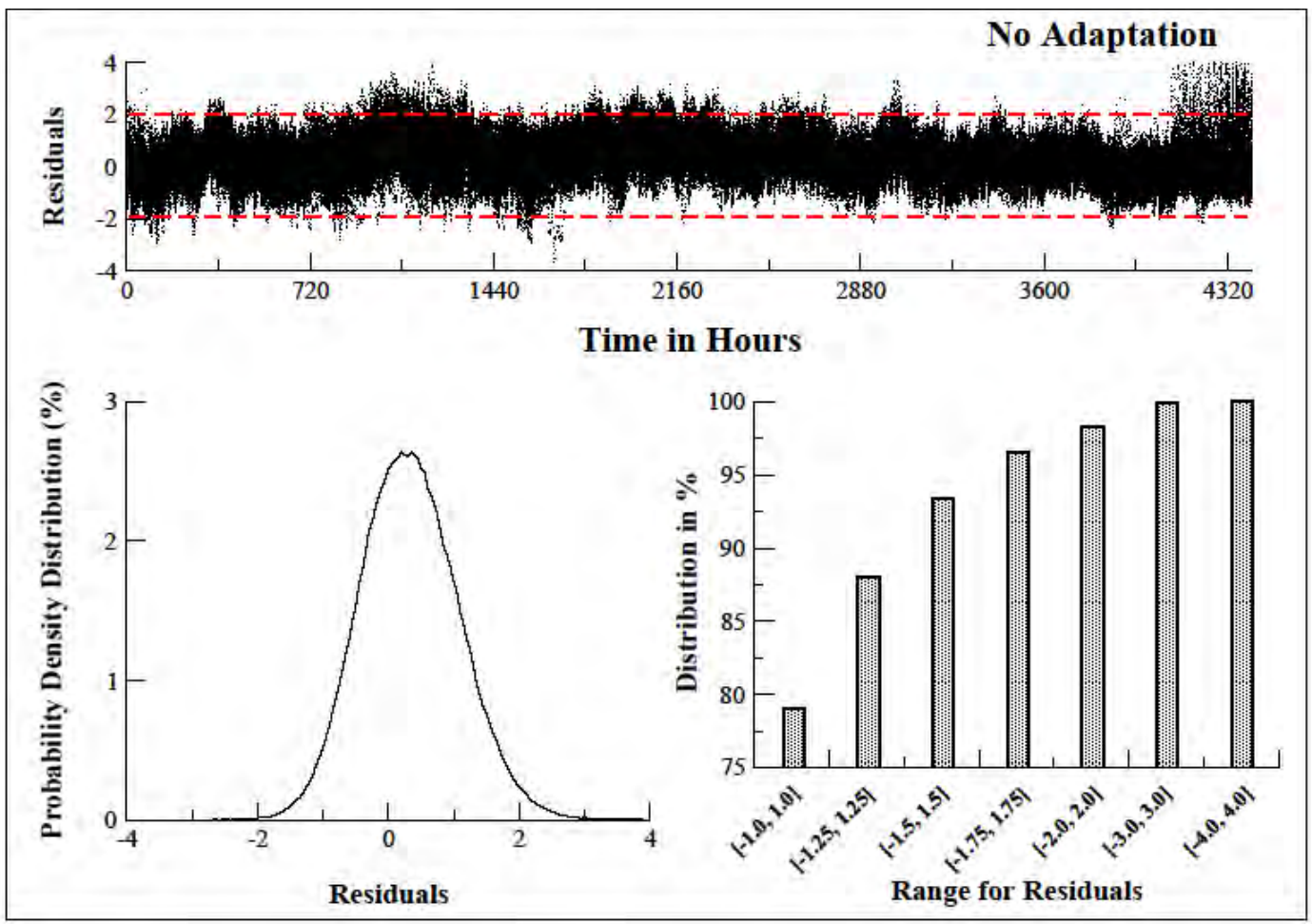

Fig. 1. Comparison of ESP-r and regression equation hourly temperatures scenarios for "no adaptation", London 2030 Medium emission scenario

In summary, Fig. 1 presents (top graph) over 400,000 data points between April and October for the specific scenario, where "residual" is the difference between the internal hourly temperature of ESP-r and the regression equation (in degrees). Due to the large amount of data, it is difficult to discern a typical error from this graph alone, hence the use of the left graph which shows that the majority of the "error" between ESP-r and the regression equation is $\pm 1^{\circ} \mathrm{C}$ - deemed an acceptable error over such a vast amount of data and for an hourly resolution. The right graph demonstrates that almost $80 \%$ of the data is within this error. The validation exercise provides an indication that an appropriately calibrated regression equation can be used to emulate a dynamic simulation over a large number of climates, providing an initial simulation has been carried out to establish the relationship in the first place.

\subsection{Design approach}

The integration of any future-climate design tool into the building design process involves an understanding of existing design practices. To investigate this, the project is running several focus groups to obtain feedback from a wide range of design professionals in the UK. These focus groups will discuss how current overheating analyses are carried out for domestic and non-domestic buildings, and how low-energy buildings might be more susceptible to future overheating for certain scenarios.

In summary, it is imagined that the methodology discussed in sections 2.1 to 2.3 might be used as follows:

1. A building is designed to current building regulations with an overheating analysis based on dynamic simulation of, nominally, a current hourly climate file

2. The proposed regression tool, working in parallel with the simulation engine for step 1 , generates a series of regression coefficients based on the documented principal component analysis framework (see section 2.3) 
3. A random selection of 100 climate years for a specific future scenario can be selected from the UKCP'09 database (e.g. the user would choose: London, medium emission, 2020-2049) - this can be integrated into the tool so that the user would not need to access the climate information separately. These climates will not need to be simulated through the dynamic building software

4. The user chooses an overheating metric, such as the percentage of hours over $28^{\circ} \mathrm{C}$ or another defined threshold, that is suitable for that building type

5. The regression tool provides an overheating risk output, demonstrating the probability of different scales of overheating for that building in a future climate (see section 3 for examples)

If a building is designed to achieve adequate thermal comfort for a current climate, the above methodology can estimate whether that same building will meet thermal comfort criteria for chosen future climates.

\section{Results}

The project is looking at a selection of buildings, two of which will be used below to demonstrate the way that the analysis described in section 2 can be used to quantify the effect of adaptation scenarios to prevent overheating in a naturally ventilated building. The technique is also being applied to mechanically cooled buildings, with results forthcoming.

\subsection{Domestic building}

The domestic building case study is designed to represent a typical UK 3-bedroom dwelling, with infiltration rate of $0.7 \mathrm{ac} / \mathrm{h}$, with wall $\mathrm{U}$-value of $0.37 \mathrm{~W} / \mathrm{m}^{2} \mathrm{~K}$. Detail of the construction and internal activity can be found in previous publications [7]. Fig. 2 shows the simulation diagram used by ESP-r.

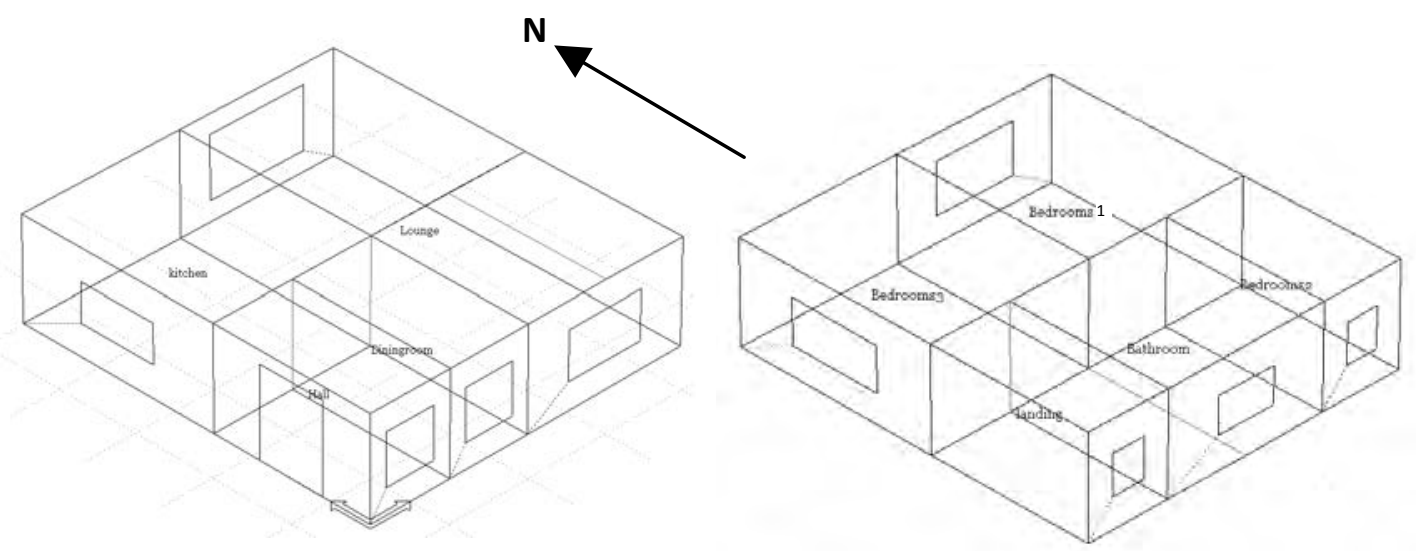

Fig. 2. ESP-r diagram of ground floor (left) and first floor (right) of modelled dwelling

While a large range of overheating criteria could be specified for this building type, the chosen metric for this paper is the number of hours in the bedroom that exceed $23.9^{\circ} \mathrm{C}$ at night. This definition, justified elsewhere [7], proposes that the lack of options to adapt to overheating at night may cause an occupant to take other measures (e.g. purchase a domestic air-conditioning unit) to provide an improved level of thermal comfort. The building is simulated for all the climate scenarios identified in section 2.1, and for three adaptation scenarios, applied cumulatively: i) "no adaptation", where the occupant does not react to overheating at all; ii) "window opening", where windows are opened in the bedroom zones if that zone exceeds $23.9^{\circ} \mathrm{C}$ (and closed if the temperature then drops below); iii) "external shading and reduced internal gains”, where horizontal slats are placed above every window to 
reduce solar gain, while internal heat gains (from appliances and lighting) are reduced by $25 \%$ to represent more efficient technologies. For simplicity, Fig. 3 only shows the results for the 2030s medium emission scenario for a London location, though the same format can be applied to any climate scenario. The $\mathrm{x}$-axis of the graph shows relative change in the overheating metric (i.e. number of hours above the $23.9^{\circ} \mathrm{C}$ threshold at night) against a 1960 1990 baseline. With 100 equally probable climate-years used for this future climate scenario, it is possible to construct a cumulative frequency plot that suggests the probability of different levels of overheating occurring. The effect of the adaptation scenarios is clear, with the overheating risk curves being morphed in the negative direction on the $\mathrm{x}$-axis - representing reduced overheating risk. Such a graph could be used to design a building to be sensitive to a future climate: for example, the "no adaptation" scenario estimates a $96 \%$ probability of more overheating (i.e. the part of the cumulative curve that is to the right of the "zero" dotted line representing no change) for the future climate used. Applying both adaptation scenarios reduces this to just $14 \%$; i.e. the building now has just a $14 \%$ chance of being warmer in the future. This may be a suitably low future overheating risk, providing the client with the confidence that their building should provide adequate thermal comfort in the future.

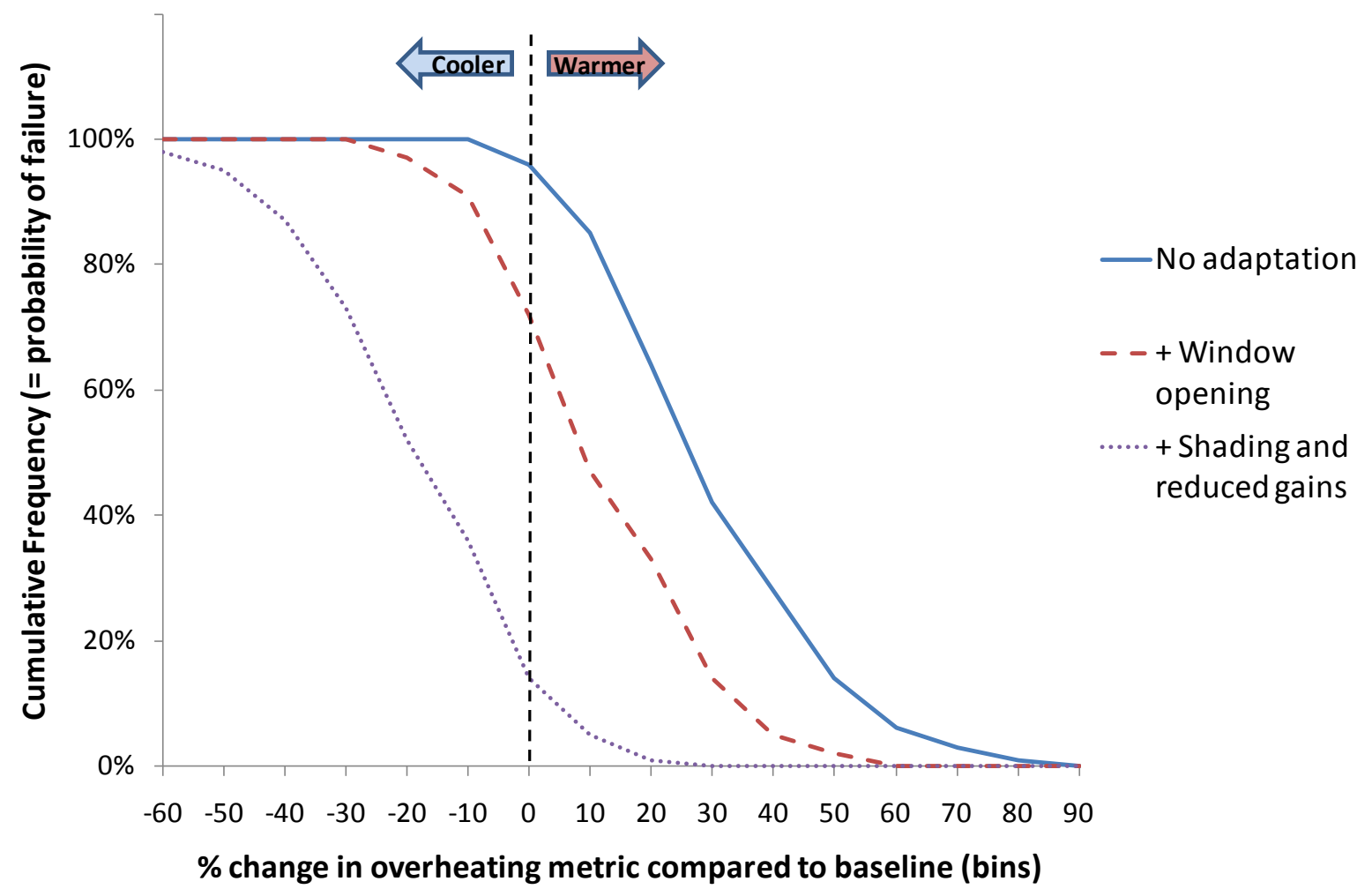

Fig. 3. Predicted increase in dwelling overheating for $100 \mathrm{r}$ andom climates for London, Medium Emission, 2030 scenario

\subsection{Primary School}

The exercise is repeated for a primary school building, previously analysed with deterministic climates in the Tarbase project [8]. The construction and internal activity is specified in detail in this aforementioned reference and relates to UK Building Regulations for the assumed build date of 2000. The overheating criterion used for this building is the percentage of hours above $28^{\circ} \mathrm{C}$ in teaching areas, as suggested by UK building guides [10]. As with the previous case-study, the methodology of section 2 is carried out to assess future overheating risks. Fig. 4 summarises the floor plan and building design. 


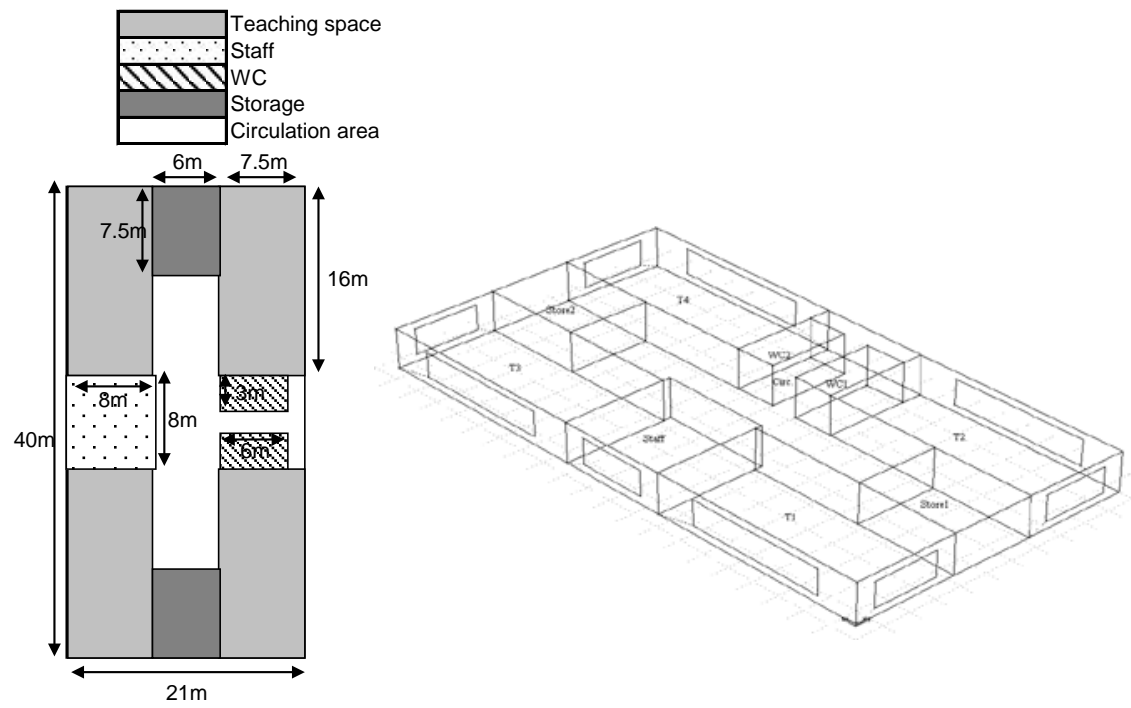

Fig. 4. Layout and plan of primary school case-study

Fig. 5 demonstrates the predicted overheating curves, again for the 2030s medium emission scenario for a London location. The adaptation scenarios are: i) "no adaptation"; ii) "Increase Vent”, where maximum ventilation rates are increased from 81/s/person to 12l/s/person; iii) "Reduced gains", for energy-efficient appliances and lighting that reduce internal heat gains (as quantified elsewhere [8]); iv) "External shading", with simple horizontal shades added above each window. As with the domestic case study (Fig. 3), a substantial improvement is made as a result of the adaptations - ranging from a $96 \%$ chance of increased overheating for "no adaptation" to a $0 \%$ chance once all adaptations have been applied.

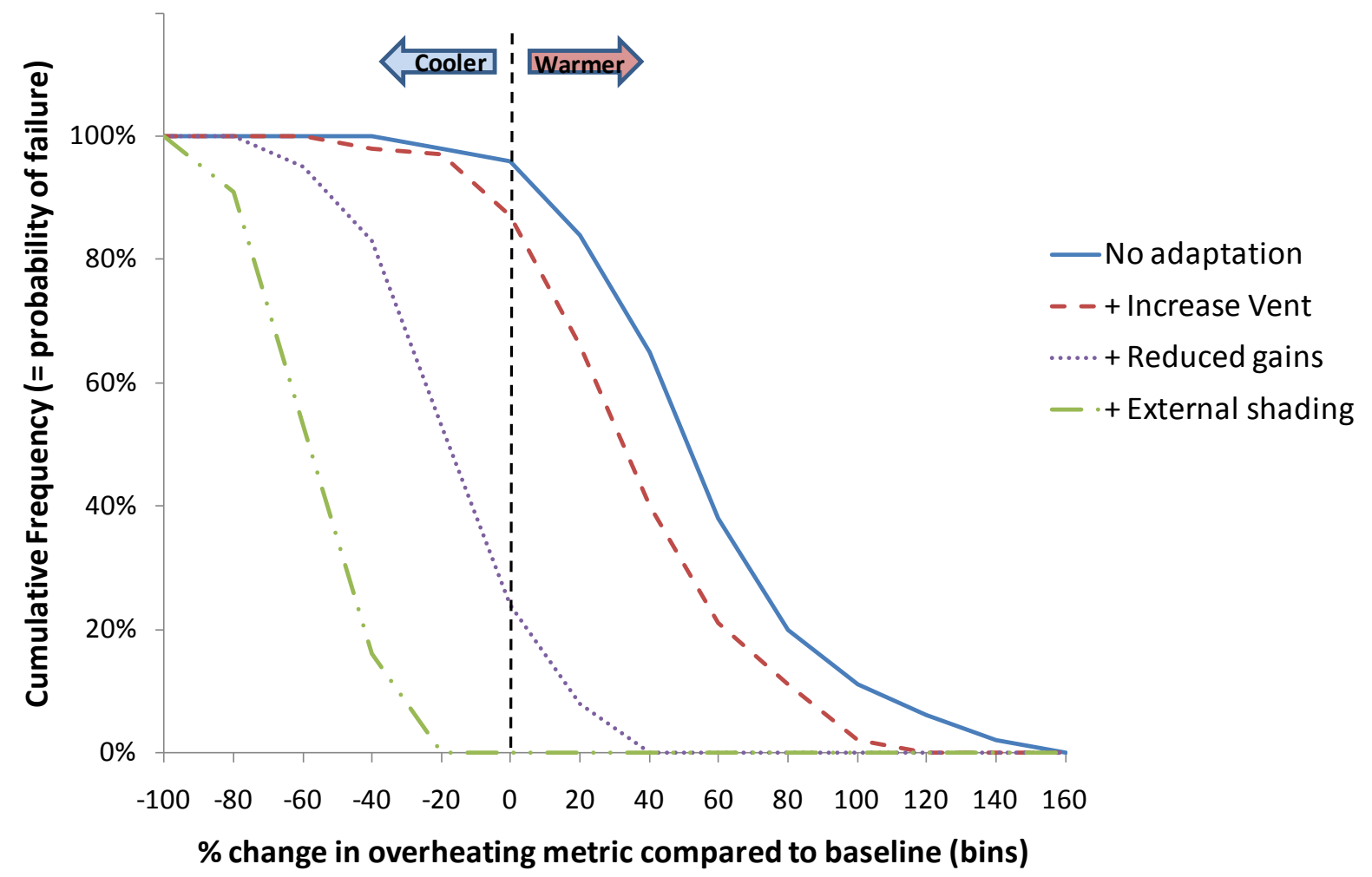

Fig. 5. Predicted increase in school overheating for 100 random climates for London, Medium Emission, 2030 scenario 


\section{Discussion and Conclusions}

The Low Carbon Futures project aims to provide guidance for designing buildings, or retrofitting existing buildings, so that they will provide adequate thermal comfort for a future climate. There are essentially two problems to be addressed: i) how can designers be encouraged to design for a future climate, rather than just using existing climate definitions and ii) if future climates are presented in a probabilistic form (as with UKCP'09), can this be integrated into the design process in an efficient way? If this latter problem provides an additional barrier to building design, then it will not be adopted in practice. However, if this form of climate projection can be linked to an overheating "risk analysis", which might have parallels with existing risk assessments that the building industry are required to carry out, then the described method might be seen as attractive to both building professional and their client. The methodology described in this paper, which produces probabilistic overheating curves for a specific building once that building has undergone a single simulation, is suitable for use in a design tool that would not require a dramatic increase in building simulation time, despite the use of hundreds of climate-years from the UKCP'09 database. The results suggest that it should be possible to find a compromise between an efficient calculation method and a reliable output that maintains the detail from the probabilistic climate projections used, though it should be stressed that the proposed tool emulates building simulation output, not empirical data. The project will subsequently be testing this approach against a wider selection of buildings and adaptation scenarios to determine if the described methodology is truly universal for future overheating analyses of buildings in the UK.

\section{References}

[1] UK Government Department of Communities and Local Government, Proposals for amending Part L and Part F of the Building Regulations, April 2010

[2] M. Holmes and J.N. Hacker, Climate change, thermal comfort and energy: Meeting the design challenges of the 21st Century, Energy and Buildings 39, 2007, pp. 802-814

[3] P.D. Jones et al., UK Climate Projections science report: Projections of future daily climate for the UK from the Weather Generator, available from http://ukclimateprojections.defra.gov.uk

[4] Adaptation and Resilience in a Changing Climate Network (ACN), Programme website http://www.ukcip-arcc.org.uk/content/view/605/519/

[5] Low Carbon Futures project summary page, http://www.ukciparcc.org.uk/images/stories/pdfs/lowcarbon_future_leaflet.pdf

[6] Carbon Vision Buildings “Tarbase” project website, http://www.tarbase.com

[7] A.D. Peacock, D.P. Jenkins and D. Kane, Investigating the potential of overheating in UK dwellings as a consequence of extant climate change, Energy Policy 38, 2010, pp. 3277-3288

[8] D.P. Jenkins, A.D. Peacock and P.F.G. Banfill, Will future low-carbon schools in the UK have an overheating problem?, Building and Environment 44, 2009, pp. 490-501

[9] S. Patidar, D.P. Jenkins, G. Gibson, and P.F.G. Banfill, Statistical techniques to emulate dynamic building simulations for overheating analyses in future probabilistic climates, Journal of Building Performance Simulation, In Press, 2010

[10]Chartered Institution of Building Services Engineers (CIBSE), Environmental Design, CIBSE Guide A, 2006 